\title{
Circulating tumor cells from a 4-dimensional lung cancer model are resistant to cisplatin
}

\author{
Monika Vishnoi, PhD, ${ }^{a}$ Dhruva K. Mishra, PhD, ${ }^{a}$ Michael J. Thrall, MD, ${ }^{b}$ Jonathan M. Kurie, $\mathrm{MD},{ }^{\mathrm{c}}$ and \\ Min P. Kim, MD, FACS ${ }^{\mathrm{a}, \mathrm{d}}$
}

Objective: To determine the effect of cisplatin on circulatory tumor cells (CTC) and tumor nodules in a four-dimensional (4D) lung cancer model.

\begin{abstract}
Methods: CTCs from the 4D model seeded with H1299, A549, or H460 and respective cells that were grown under two-dimensional conditions in a Petri dish were treated with $50 \mu \mathrm{M}$ cisplatin for 24 and 48 hours and cell viability was determined. The lung nodules in the 4D model were then treated with different continuous or intermittent doses of cisplatin and the nodule size, the number of CTCs, and the level of matrix metalloproteinase (MMP) were determined.
\end{abstract}

\begin{abstract}
Results: Cisplatin led to a significant decrease in the viability of tumor cells grown under $2 \mathrm{D}$ conditions $(P<.01)$ but not in CTCs from the 4D model after both 24 hours and 48 hours. Cisplatin led to regression of tumor nodules with both the continuous and intermittent treatments. Moreover, there was a significantly higher number of CTCs per tumor area $(P<.05)$ and MMP-2 production per tumor area $(P=.007)$ for all human lung cancer cell lines grown in the $4 \mathrm{D}$ model when treated with cisplatin.
\end{abstract}

Conclusions: The 4D lung cancer model allows for the isolation of CTCs that are resistant to cisplatin treatment. The model may allow us to better understand the biology of cisplatin resistance. (J Thorac Cardiovasc Surg 2014;148:1056-64)

Lung cancer is the most common cause of cancer-related deaths in the United States. ${ }^{1}$ The 5-year survival for patients with lung cancer has increased only incrementally, from $13 \%$ in 1975 to $16 \%$ in 2005 . $^{2}$ The reason for the high mortality and morbidity is that most patients with lung cancer who present with metastatic disease develop resistance to chemotherapy. ${ }^{1}$ One of the most effective treatments for lung cancer is platinum-based therapy, including cisplatin. Cisplatin binds to and causes cross-linking, breaks, and mutations in the DNA chain, injuries that trigger apoptosis. It provides a high initial response but most patients with

$\overline{\text { From Departments of Surgery }}{ }^{\mathrm{a}}$ and Pathology and Genomic Medicine, ${ }^{\mathrm{b}}$ Houston Methodist Research Institute, Houston, Tex; Thoracic/Head and Neck Medical Oncology, ${ }^{\mathrm{c}}$ University of Texas MD Anderson Cancer Center, Houston, Tex; and Department of Surgery, ${ }^{\mathrm{d}}$ Weill Cornell Medical College, Houston Methodist Hospital, Houston, Tex.

M.P. Kim has received grant support from the Second John W. Kirklin Research Scholarship, the American Association for Thoracic Surgery Graham Research Foundation, and the Houston Methodist Foundation with a donation from J. Michael Jusbasche.

Disclosures: Min P. Kim has received honoraria from Covidien and Ethicon for teaching a VATS lobectomy course, and has applied for a patent related to this work. All other authors have nothing to disclose with regard to commercial support. Monika Vishnoi and Dhruva K. Mishra contributed equally to this work as first author.

Read at the 94th Annual Meeting of The American Association for Thoracic Surgery, Toronto, Ontario, Canada, April 26-30, 2014.

Received for publication April 2, 2014; revisions received May 14, 2014; accepted for publication May 21, 2014.

Address for reprints: Min P. Kim, MD, FACS, 6550 Fannin St, Suite 1661, Houston, TX 77030 (E-mail: mpkim@houstonmethodist.org).

$0022-5223 / \$ 36.00$

Copyright (c) 2014 by The American Association for Thoracic Surgery

http://dx.doi.org/10.1016/j.jtcvs.2014.05.059 lung cancer eventually relapse as a result of resistance to cisplatin. ${ }^{3}$

The mechanism of chemotherapeutic resistance is difficult to study because of the lack of preclinical models that mimic metastasis. Drug sensitivity data from a twodimensional (2D) culture are often misleading, ${ }^{4}$ and in vivo mouse studies, although the gold standard for anticancer drug studies, are not always predictive of patient response. ${ }^{5}$ Lung cancer can often be cured in mice in such studies, but those results translate poorly to treating patients with lung cancer. Efforts to improve the available models for anticancer drug research have led to the development of improved in vitro models.

Recently, we have developed a novel four-dimensional (4D) lung model that forms perfusable nodules from human non-small cell lung cancer cells grown on an acellular rat matrix. Unlike other in vitro models, it maintains a separation from its vascular or endothelial space and the epithelial space in the matrix, ${ }^{6}$ allowing the tumor cells to grow in the three-dimensional space and the medium to flow through the vasculature and create a perfusable nodule. The addition of flow makes this a unique 4D model. This 4D model mimics the histopathology and protease secretion pattern of human lung cancer. ${ }^{7,8}$ Furthermore, differential gene expression profiling shows that the gene signature of tumor cells grown in the 4D model predicts poor survival, whereas the same cells grown in the 3D model or cells grown simply in a Matrigel predict good survival. ${ }^{9}$ This suggests that the 4D model allows tumor cells to behave in a more aggressive fashion. 

Abbreviations and Acronyms
$2 \mathrm{D}=2$ dimensional
$4 \mathrm{D}=4$ dimensional
CTC $=$ circulatory tumor cell
$\mathrm{MMP}=$ matrix metalloproteinase

As the tumor nodules form in the 4D model, we have observed live tumor cells in the circulating medium or circulating tumor cells (CTCs). We postulated that the 4D model would show the impact of cisplatin on the primary tumor, the number of CTCs, and protease secretion.

\section{MATERIALS AND METHODS}

The Institutional Animal Care and Use Committee at the Methodist Hospital Research Institute approved all the animal experiments in our study.

\section{Cell Culture}

American Type Culture Collection (ATCC, Manassas, Va) human non-small cell lung cancer cell lines H1299, A549, H460, and HCC827 were used. The $\mathrm{H} 1299$ cell line has a p53 deletion and was derived from lymph node metastasis; the A549 cell line was derived from the primary adenocarcinoma of the lung; the $\mathrm{H} 460$ cell line was derived from the pleural fluid of a patient with large cell lung cancer; and the HCC827 cell line has a mutation in the EGFR tyrosine kinase domain and was derived from primary lung adenocarcinoma. The cells were grown in cell culture flasks (BD Biosciences, San Diego, Calif) in complete medium made from RPMI 1640 (HyClone, Logan, Utah) supplemented with $10 \%$ fetal bovine serum (Lonza, Basel, Switzerland) and antibiotics (100 IU/mL penicillin, $100 \mu \mathrm{g} / \mathrm{mL}$ streptomycin, and $0.25 \mu \mathrm{g} / \mathrm{mL}$ amphotericin B; MP Biomedicals, Santa Ana, Calif) at $37^{\circ} \mathrm{C}$ in $5 \% \mathrm{CO}_{2}$.

\section{D Lung Cancer Model}

The 4D lung model was created as described previously. ${ }^{7}$ Decellularized lung matrices from 6-week-old Sprague-Dawley rats (Harlan, Houston, Tex) were used and 25 million $\mathrm{H} 1299,{ }^{10} \mathrm{~A} 549,{ }^{11} \mathrm{H} 460,{ }^{10}$ or HCC $827^{12}$ human lung cancer cells (ATCC) diluted in $50 \mathrm{~mL}$ of complete medium were seeded in the trachea of the ex vivo 4D lung model. A bioreactor was set up with $200 \mathrm{~mL}$ of complete medium as described previously. ${ }^{7,8}$ The medium goes through the pulmonary artery of the acellular lung, perfuses the lung, exits the lung, and collects in a reservoir, where it is pumped in a closed circuit at $6 \mathrm{~mL}$ per minute, and an oxygenator before it is returned to the acellular lung. Medium was collected from the reservoir every day. Conditioned medium was spun at $500 \times g$ for 5 minutes and the cells were diluted in $1 \mathrm{~mL}$ of complete medium. The number of live tumor cells were counted using an automatic cell counter (TC20 cell counter; Bio-Rad, Hercules, Calif). These cells were labeled as the CTCs. The medium in the bioreactor was replaced with fresh complete medium daily.

\section{Cisplatin Treatment of CTCs and 2D Cells}

Ten thousand 10,000 CTCs from the 4D model seeded with H1299, A549, or H460 or respective cells that were grown on a Petri dish (2D) were seeded in 96-well plates in $200 \mu \mathrm{L}$ of complete medium. HCC827 cells were excluded from this experiment because we could not reliably count the number of cells using the automatic cell counter because of cell clumping. Half of the plated CTCs or $2 \mathrm{D}$ cells were treated with $50 \mu \mathrm{M}$ cisplatin $(\mathrm{n}=12)$ and the other half were used as a control group $(\mathrm{n}=12)$. The number of live cells in the supernatant and live cells that were adherent to the plate after trypsinization with $0.25 \%$ trypsin (HyClone) were counted at 24 hours $(n=6)$ or 48 hours $(n=6)$. The number of total live cells (the sum of the live cells in the supernatant and the live cells adherent to the plate) were compared between the CTCs or 2D cells that were treated with cisplatin and the control group.

\section{Cisplatin Treatment of Tumor Nodules Grown on the 4D Model}

The 4D lung cancer model was seeded with H1299 and grown for 14 days $(\mathrm{n}=9)$. The $4 \mathrm{D}$ model was seeded with no cisplatin (the control group, $\mathrm{n}=3)$, cisplatin at $10 \mu \mathrm{M}(\mathrm{n}=3)$, or $50 \mu \mathrm{M}(\mathrm{n}=3)$ starting on day 4 after seeding of the nodule. The lung tissue of the 4D lung model was examined for the presence of nodules every other day. The area per nodule was calculated and added to obtain the total nodule size. On days 8,11 , and 14 , a right upper lobectomy, a right middle lobectomy, and a right lower lobectomy were performed, respectively. The lung tissues from the ex vivo 4D lung model from the lobectomy were processed as described previously ${ }^{7}$ and evaluated by a board-certified pathologist (M.T.). To confirm the results from hematoxylin and eosin staining and to determine cell proliferation and cell death, immunohistochemical analysis was performed on lung tissue from day 8 in 2 groups (the control group and the 50- $\mu \mathrm{M}$ treatment group). For the immunohistochemical analysis, the standard protocol was followed as previously described. ${ }^{8}$ The percentage of Ki-67 and caspase- 3 staining was determined by examining 5 random high-power fields. CTCs were isolated and counted daily as described earlier. Next, the number of CTCs per tumor area was determined by dividing the number of CTCs by the size of the tumor from each given day.

In addition, the left side of the 4D lung cancer model was seeded with either H1299, A549, H460, or HCC827 and treated with the physiologic concentration of cisplatin observed in the plasma in patients ${ }^{13}$ after treatment with $10 \mu \mathrm{M}$ cisplatin given weekly in 3 doses starting on day 4 for 21 days $(n=4)$ or untreated as a control $(n=4)$. The $4 D$ lung cancer model was examined for the presence of nodules every 5 days. The area per nodule was calculated and added to obtain the total nodule size. The number of CTCs per tumor area was also calculated. The area under the curve for tumor size and CTCs per tumor area was measured for the model treated with cisplatin and the control group. The area under the curve for tumor size and CTCs per tumor area was compared for the control group and cisplatin-treated group.

\section{Matrix Metalloproteinase Levels in the Medium}

A matrix metalloproteinase (MMP) assay was performed using the medium collected from the 4D lung models treated with an intermittent concentration of cisplatin $(10 \mu \mathrm{M})$ as described earlier. A MILLIPLEX Human MMP Panel 2 kit (Millipore, Billerica, Mass) was used to detect both active and inactive MMP-1, MMP-2, MMP-7, MMP-9, and MMP-10 following the manufacturer's protocol. $^{8}$ A Luminex 200 instrument was used to read the plate. The results were further analyzed using MILLIPLEX Analyst Software (Millipore). The MMP level per tumor area was calculated and the area under the curve was determined for the model treated with cisplatin and the control group. The results were then normalized to the control group and the average MMP level per tumor area was determined. The normalized area under the curve of MMP per tumor area was compared for all 3 cell lines.

\section{Statistical Analysis}

Independent 2-sample $t$ tests were performed to identify significantly different mean number of live cells after cisplatin treatment of CTCs and $2 \mathrm{D}$ cells. In addition, a $t$ test was used to test for mean tumor size on different days for continuous cisplatin treatment and significantly different percentages of Ki-67 staining and percentages of caspase- 3 staining on day 8. Moreover, $t$ tests were done to test for the number of CTCs per tumor 


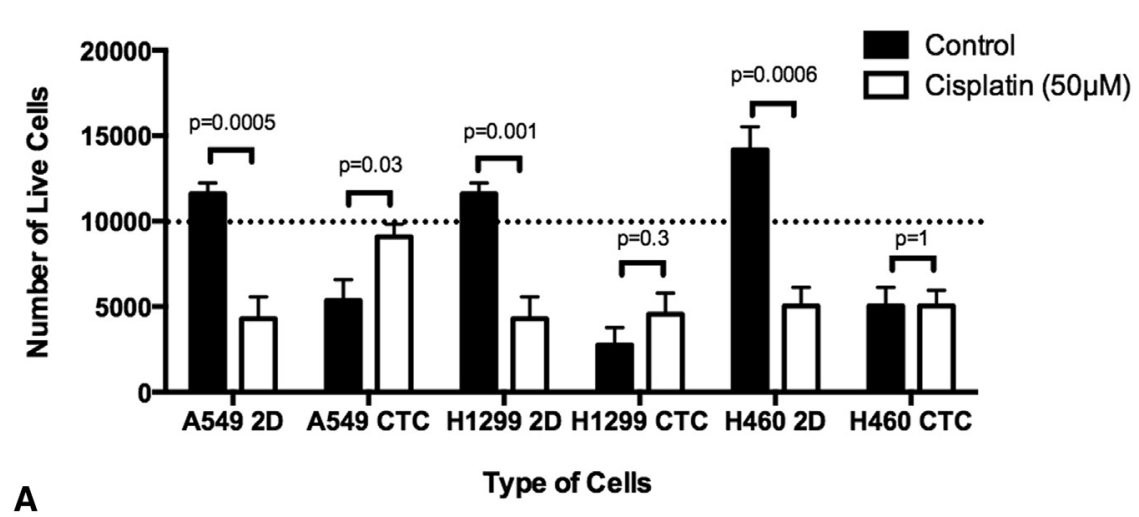

A

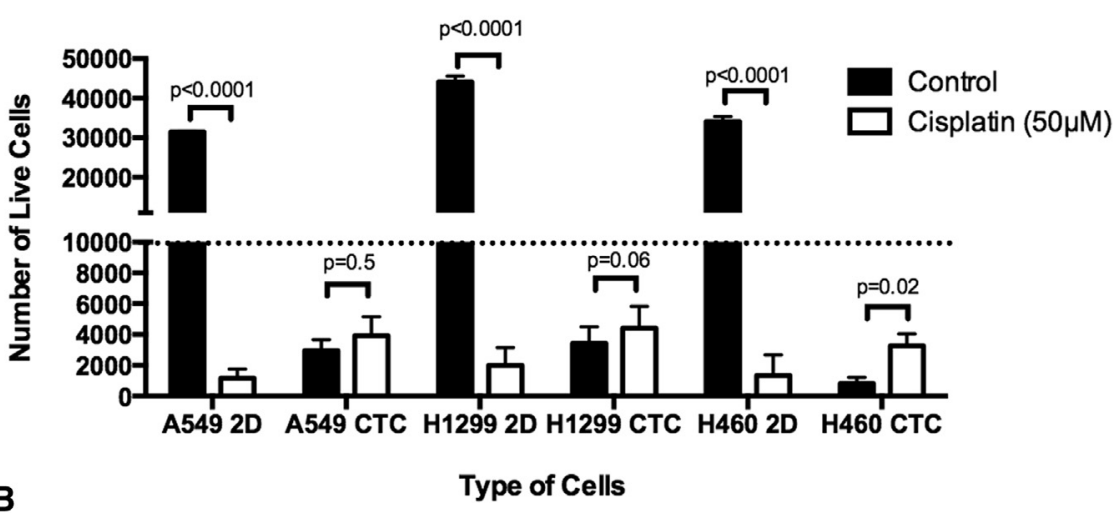

FIGURE 1. Cisplatin response of CTCs from the 4D model and 2D cells. A and B, The bar diagram shows the number of live tumor cells found after 24 hours (A) or 48 hours (B) of treatment of A549, H1299, and H460 2D cells and CTC cells with $50 \mu \mathrm{M}$ cisplatin. The CTCs showed no significant decrease in cell viability compared with the control group, whereas the 2D cells showed significantly fewer cells than the control group. CTC, Circulatory tumor cells.

area between the continuous cisplatin-treated $(50 \mu \mathrm{M}$ or $10 \mu \mathrm{M}) 4 \mathrm{D}$ model and the control group and the area under the curve for tumor size and the area under the curve for CTCs per tumor area for the intermittent treatment of cisplatin $(10 \mu \mathrm{M})$ compared with the control group. A $t$ test was used to compare the normalized area under the curve of MMP per tumor area. All analyses were performed using PRISM Version 5.0 software (GraphPad Software, La Jolla, Calif).

\section{RESULTS}

\section{Circulating Tumor Cells From the 4D Model}

There were significantly fewer total live tumor cells when tumor cell lines grown on Petri dishes (2D) were treated with $50 \mu \mathrm{M}$ cisplatin for 24 hours (Figure $1, A$ ) or 48 hours (Figure $1, B$ ) compared with the control group. There were significantly fewer A549 2D $(P=.0005), \mathrm{H} 1299$ 2D $(P=.001)$, and H460 2D $(P=.0006)$ when these cells were treated with cisplatin for 24 hours after plating on a Petri dish as well as when A549 2D $(P<.0001)$, H1299 2D $(P<.0001)$, and H460 2D $(P=.02)$ were treated with cisplatin for 48 hours. At both time points, the control 2D cells had a greater number of cells in the cultured condition than the cells that were plated (dotted line).

On the other hand, there was no significant decrease in the total live tumor cells in CTCs from the 4D model that were treated with $50 \mu \mathrm{M}$ cisplatin for 24 hours (Figure 1, A) or 48 hours (Figure 1,B) on a Petri dish compared with the control group. For A549 CTCs, there were significantly more live tumor cells when treated with cisplatin compared with the control group $(P=.03)$ at 24 hours although this difference disappeared at 48 hours $(P=.5)$. For H1299 CTCs, there was no significant difference in the number of cells when treated with cisplatin for both 24 hours $(P=.3)$ and 48 hours $(P=.06)$ compared with the control group. For H460 CTCs, there was no significant difference in the number of live tumor cells after 24 hours of cisplatin treatment $(P=1)$ but after 48 hours there were significantly more tumor cells with cisplatin treatment compared with the control group $(P=.02)$. Moreover, at both time points the control CTC cells had fewer cells in the cultured condition compared with the number of cells that were plated (dotted line).

\section{Continuous Cisplatin Treatment of Cells in the 4D Model}

In the $4 \mathrm{D}$ lung model, $97.6 \% \pm 4.9 \%$ of all cells lines adhered to the decellularized lung matrix after 3 passes and incubation for 2 hours. The H1299 cells formed nodules on the 4D lung model by day 4 (Figure 2). The tumor nodules enlarged without cisplatin treatment on day 4 


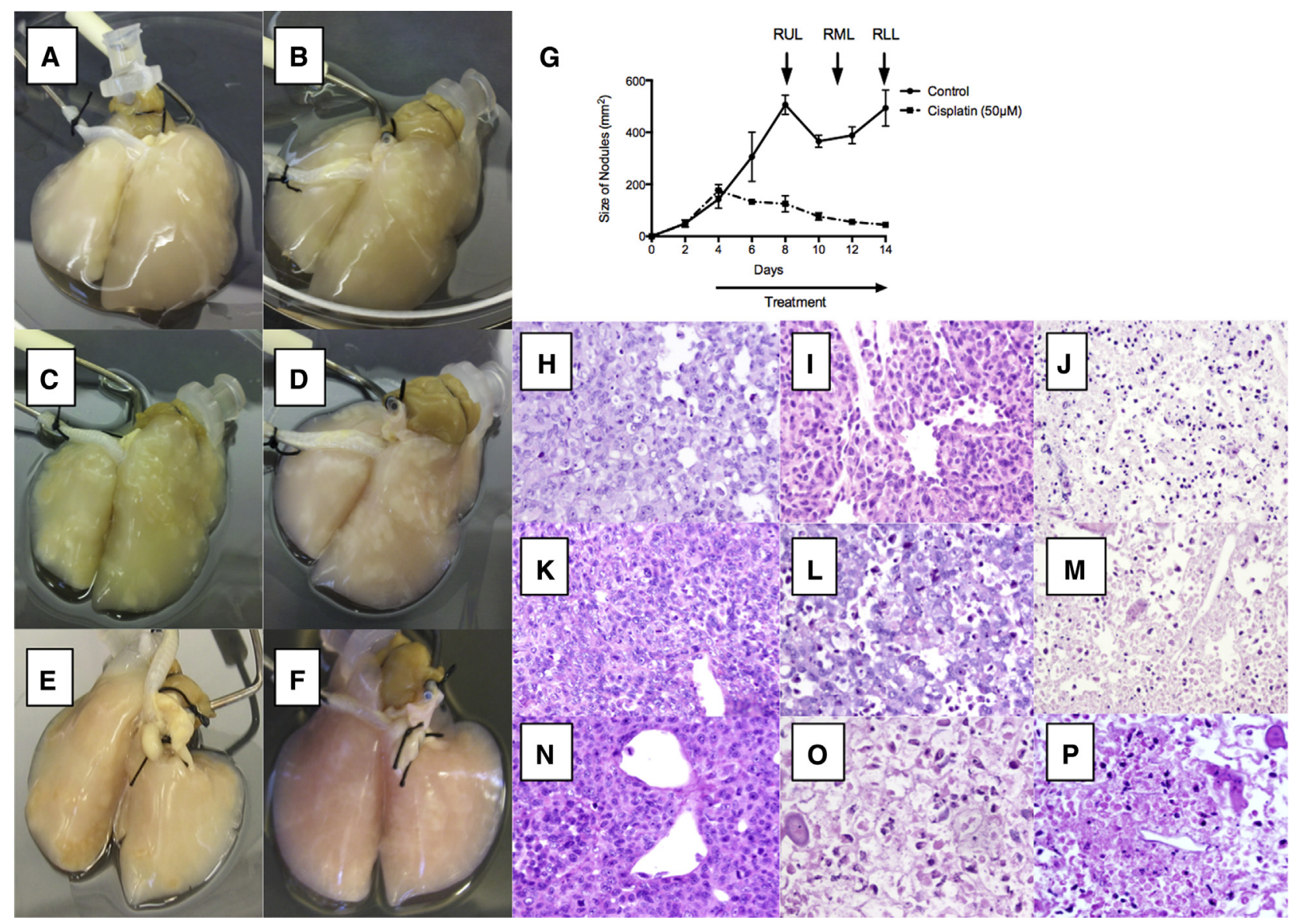

FIGURE 2. Cisplatin leads to tumor nodule regression in the 4D model. A and B, Tumor nodules formed on the 4D model before the start of treatment with no significant difference in the size of the nodules on day 4. C and D, On day 8 with continual tumor growth in the control group (C); there is no growth of the nodule for the 4D model treated with cisplatin $(50 \mu \mathrm{M})(\mathrm{D})$. E and F, On day 11 showing the continuous growth of a tumor nodule in the control 4D model (E); there is regression of the tumor nodule with cisplatin $(50 \mu \mathrm{M})$ treatment $(\mathrm{F})$. G, The graph shows significant nodule regression with $50 \mu \mathrm{M}$ cisplatin treatment compared with the control group. The total nodule size did not differ before treatment on day 4. H-P, Hematoxylin and eosin staining of lung tissues obtained on days 8,11 , and 14 from the control group and cisplatin-treated (10 $\mu \mathrm{M}$ or $50 \mu \mathrm{M})$ cells grown on the ex vivo 4D lung model. H-J, On day 8 , healthy tumor cells grew in the control group $(\mathrm{H})$ and cisplatin $(10 \mu \mathrm{M})$ group $(\mathrm{I})$, whereas all the cells were necrotic in the cisplatin $(50$ $\mu \mathrm{M})$ group (J). K-M, On day 11 , there are healthy cells in the control group (K) and necrotic cells in the cisplatin (50 $\mu \mathrm{M})$ group (M), whereas $50 \%$ of the cells in the cisplatin $(10 \mu \mathrm{M})$ group died $(\mathrm{L})$. N-P, On day 14, healthy tumor cells remain in the control group (N), whereas most of the cells in cisplatin $(10 \mu \mathrm{M})$ group $(\mathrm{O})$ and cisplatin $(50 \mu \mathrm{M})$ group $(\mathrm{P})$ died. $R U L$, Right upper lobe resection; $R M L$, right middle lobe resection; $R L L$, right lower lobe resection.

(Figure 2, A), day 8 (Figure 2, C), and day 14 (Figure 2,E), whereas tumor nodules regressed with continuous daily treatment with $50 \mu \mathrm{M}$ cisplatin starting on days 4 (Figure 2, B), 8 (Figure 2, D), and 14 (Figure 2, F) as well as continuous daily treatment with $10 \mu \mathrm{M}$ cisplatin (Figure 2, G). Before cisplatin treatment on day 4, the nodule size did not significantly differ between the groups (Figure $2, A$ and $B ; P=.2$ ). On day 8 , however, the nodules in the control group $\left(506 \pm 63 \mathrm{~mm}^{2}\right)$ were significantly larger than those in the groups treated with $10 \mu \mathrm{M}$ cisplatin $\left(112 \pm 34 \mathrm{~mm}^{2} ; P=.0007\right)$ and $50 \mu \mathrm{M}$ cisplatin $(125 \pm 55$ $\mathrm{mm}^{2} ; P=.0014$ ) (Figure 2). In both treatment groups, the nodules were significantly smaller on day 14 than they were on day $4(P=.001$ for $10 \mu \mathrm{M}$ cisplatin, $P=.006$ for $50 \mu \mathrm{M}$ cisplatin).
Hematoxylin and eosin analysis of the lobectomy specimens from days 8 (Figure 2, $H$ ), 11 (Figure 2, $K$ ), and 14 (Figure 2, $N$ ) from the control H1299 cells grown on the ex vivo 4D lung model showed tumor cells with cell-cell and cell-matrix interactions and with an intact vascular space without any signs of cell necrosis. However, most of the H1299 cells on the ex vivo model treated continuously with $50 \mu \mathrm{M}$ cisplatin were dead, resulting in either ghost cells without visible residual nuclear material or cells with pyknotic nuclear remnants on days 8 (Figure 2, $J$ ), 11 (Figure 2, $M$ ), and 14 (Figure 2, $P$ ). The outlines of the ghost cells showed that before treatment, the tumor cells had a growth pattern and degree of cellularity similar to those in the control group. The H1299 cells treated continuously with $10 \mu \mathrm{M}$ cisplatin showed viable tumor cells on day 8 

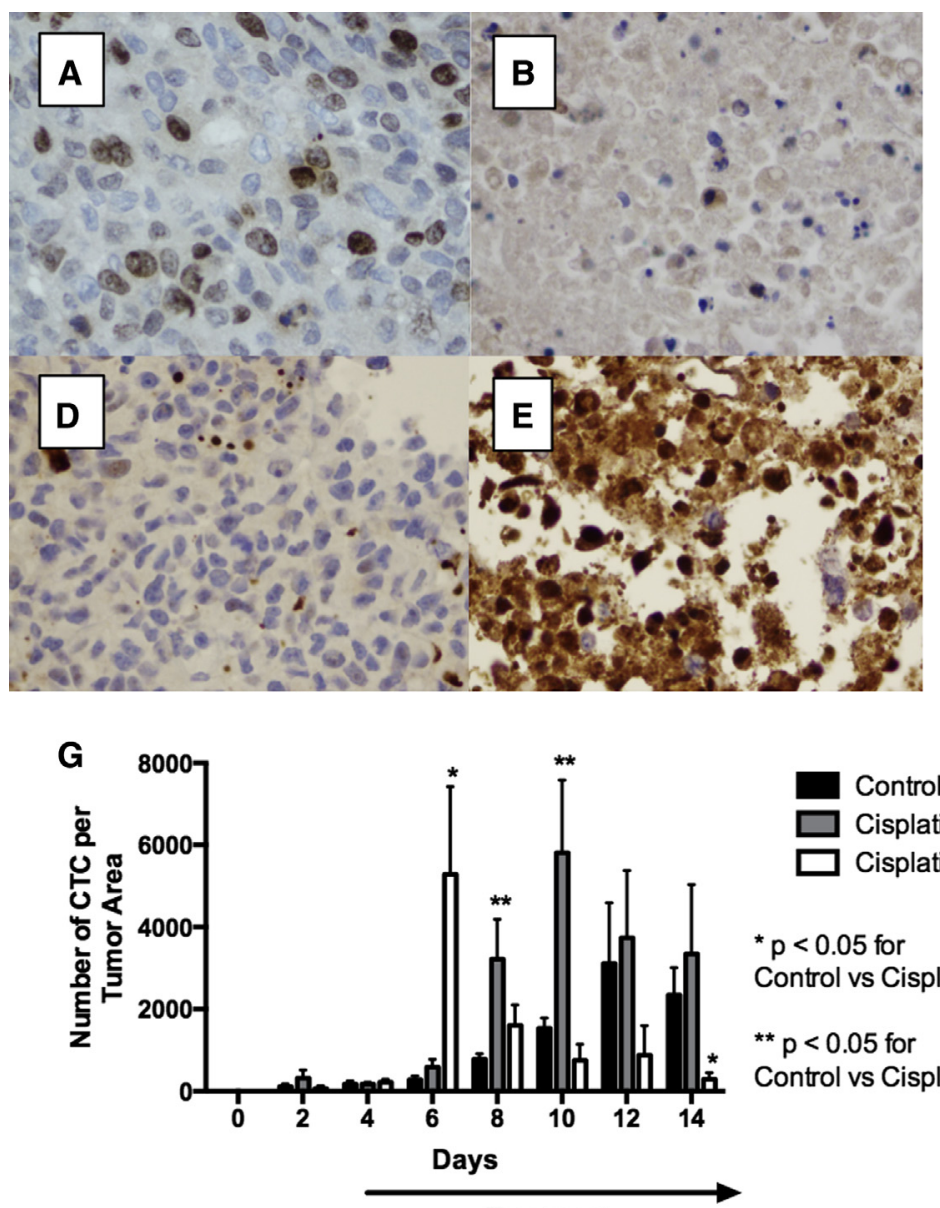

Treatment
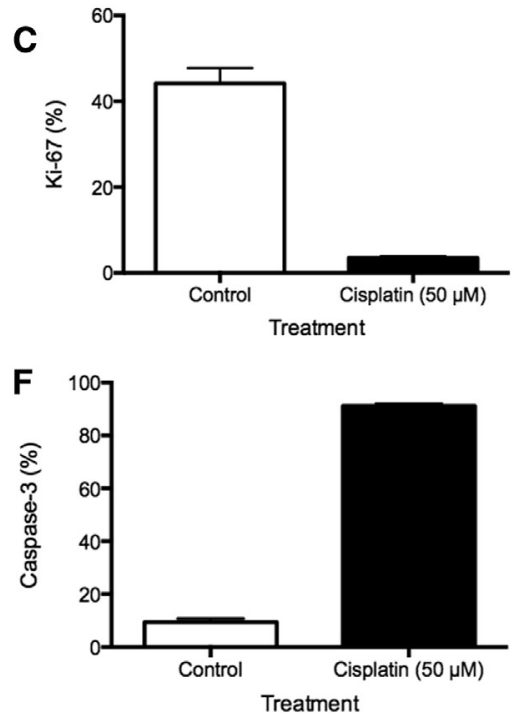

FIGURE 3. Impact of cisplatin on tumor nodules and circulatory tumor cells (CTC) production in the 4D model. A-C, Immunohistochemical analysis showed more Ki-67 staining of cells in the control group $(44 \%)$ compared with the cisplatin $(50 \mu \mathrm{M})$ group $(4 \%, P<.001)$. D-F, Immunohistochemical analyses showed a higher percentage of cells in the cisplatin $(50 \mu \mathrm{M})$ group $(91 \%)$ stained for caspase- 3 than in the control group $(9 \%, P<.0001)$. $\mathrm{G}$, Cisplatin treatment $(10 \mu \mathrm{M}$ and $50 \mu \mathrm{M})$ led to an increased number of CTCs per tumor area.

(Figure 2, I); however, on day 11, about $50 \%$ of the cells were necrotic (Figure 2, $L$ ), and on day 14, most of the cells had died (Figure 2, $O$ ).

These tissues were analyzed further for cell proliferation (Ki67) and apoptosis (CASP3). On day 8, the percentage of cells with Ki-67 staining (Figure 3, C) was much higher in the control H1299 cells grown on the ex vivo 4D lung model (Figure 3, A, Control, 44.2\% $\pm 3.6 \%$ ) compared with the cells treated continuously with $50 \mu \mathrm{M}$ cisplatin (Figure 3, $B, 3.5 \% \pm 0.4 \%, P<.0001)$. Moreover, on day 8 , most of the H1299 cells on the ex vivo model treated continuously with $50 \mu \mathrm{M}$ cisplatin stained for caspase-3 (Figure $3, E, 91.2 \% \pm 0.9 \%$ ), whereas only a few cells in the control group stained for caspase-3 (Figure 3, D, $9.4 \% \pm 1.4 \%$ ). A significantly higher percentage of H1299 cells on the ex vivo 4D lung model in the group treated continuously with $50 \mu \mathrm{M}$ cisplatin stained positive for caspase-3 than in the control group (Figure 3,F, $P<.0001)$. Further comparison of CTCs from the untreated versus the treated 4D model with H1299 showed a significantly higher number of CTCs per tumor area on day 6 with $50 \mu \mathrm{M}$ cisplatin treatment and on day 8 and 10 with $10 \mu \mathrm{M}$ cisplatin $(P<.05$, Figure $3, G)$ compared with the control group.

\section{Intermittent Cisplatin Treatment of Cells in the 4D Model}

Physiologic intermittent treatment with cisplatin $(10 \mu \mathrm{M})$ of the 4D model seeded with H1299, A549, H460, or HCC 827 cells was performed on days 4, 11, and 18. The area under the curve for the tumor nodule size for the 4D model seeded with H1299 cells treated with cisplatin was smaller compared with the control group (Figure 4, A). The average area under the curve for tumor size for all 4 cell lines treated with cisplatin was significantly smaller compared with the control group $(P=.01$, Figure $4, B)$. The area under the curve for CTCs per tumor area for the 4D model seeded with H1299 was larger compared with 


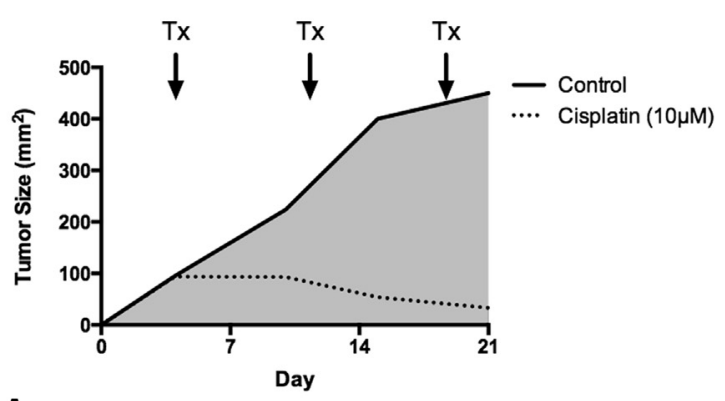

A

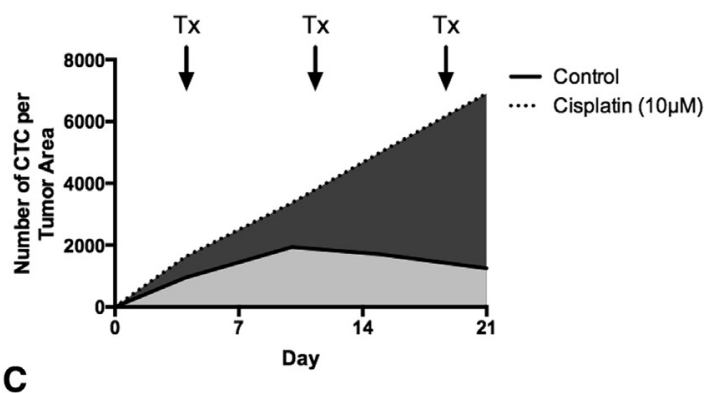

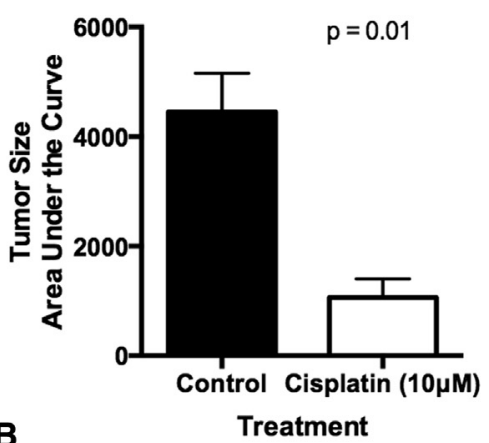

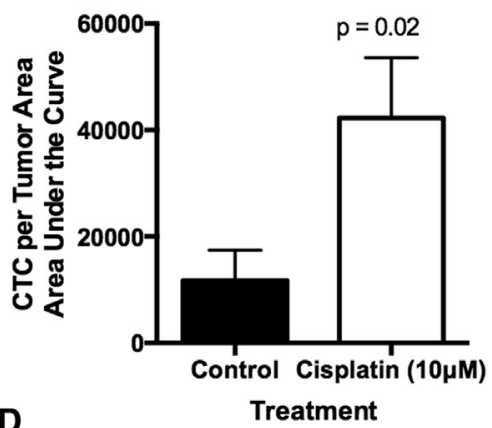

D

FIGURE 4. Tumor growth and circulatory tumor cell (CTC) production per tumor area in the 4D model with cisplatin treatment. A, Graph of tumor nodule size on the 4D lung model seeded with H1299 cells treated on days 4, 11, and 18 with $10 \mu \mathrm{M}$ cisplatin compared with the control group. B, The average area under the curve for tumor nodule size for the 4D lung model seeded with H1299, A549, H460, or HCC 827 cells treated with $10 \mu \mathrm{M}$ cisplatin is significantly smaller compared with the control group $(P=.01)$. C, Graph of the number of CTCs per tumor area of the 4D lung model seeded with H1299 cells. D, The average area under the curve for CTCs per tumor area for the 4D lung model seeded with H1299, A549, H460, or HCC827 cells shows significantly more CTCs per tumor area with $10 \mu \mathrm{M}$ cisplatin treatment compared with the control group $(P=.02)$. $T x$, Treatment.

the control group (Figure 4,C). The average area under the curve for CTCs per tumor area for all 4 cell lines treated with cisplatin was significantly higher compared with the control group $(P=.02$, Figure $4, D)$.

\section{MMPs in the Medium of the 4D Model}

Luminex analysis detected MMP-1, MMP-2, MMP-7, MMP-9, and MMP-10 in the medium of all 4 cells grown on the ex vivo 4D lung model. The area under the curve for MMP-1 per tumor area (Figure 5, $A$ ), MMP-2 per tumor area (Figure 5, $B$ ), MMP-9 per tumor area (Figure 5, D), and MMP-10 per tumor area (Figure 5,E) were higher for the 4D model seeded with H1299 treated with cisplatin compared with the control group; there was no large difference for MMP-7 per tumor area (Figure 5,C). The MMP-2 level per tumor area normalized to the control value for the 4D model seeded with 3 different cell lines showed a significant increase compared with the control group $(P=.007)$; there was no significant difference for the other 4 MMPs among the 3 cell lines (Figure 5, F).

\section{DISCUSSION}

Drug resistance is a major hurdle in cancer treatments, and can result in a poor clinical prognosis. In this study, we showed that CTCs from our 4D model are resistant to cisplatin. For 3 different human non-small cell lung cancer cell lines, cisplatin treatment did not affect this unique group of cells, although the same cells grown on a Petri dish (2D) had significantly less viable tumor cells after cisplatin treatment. This is the first time that an in vitro model has isolated a resistant group of cells without using cisplatin. Although there are several studies that show the development of cisplatin-resistant cell lines by gradually increasing the concentration and ultimately selecting a live population of cells ${ }^{14}$ to provide an understanding of the mechanism involved in drug resistance, our model improves the selection of resistant cells as they develop from a natural matrix environment as a natural phenomenon. Furthermore, the untreated CTCs showed no signs of proliferation. In the conditioned medium, the CTCs for all 3 cell lines did not proliferate. Thus, a possible mechanism for cisplatin resistance is cell cycle arrest that makes cisplatin ineffective. ${ }^{15}$

As expected, the continuous treatment of the tumor nodules formed in the 4D model seeded with H1299 cells showed a significant decrease in the primary tumor nodule size. This correlates with tumor regression in some patients with lung adenocarcinoma. ${ }^{16}$ On a computed tomography 

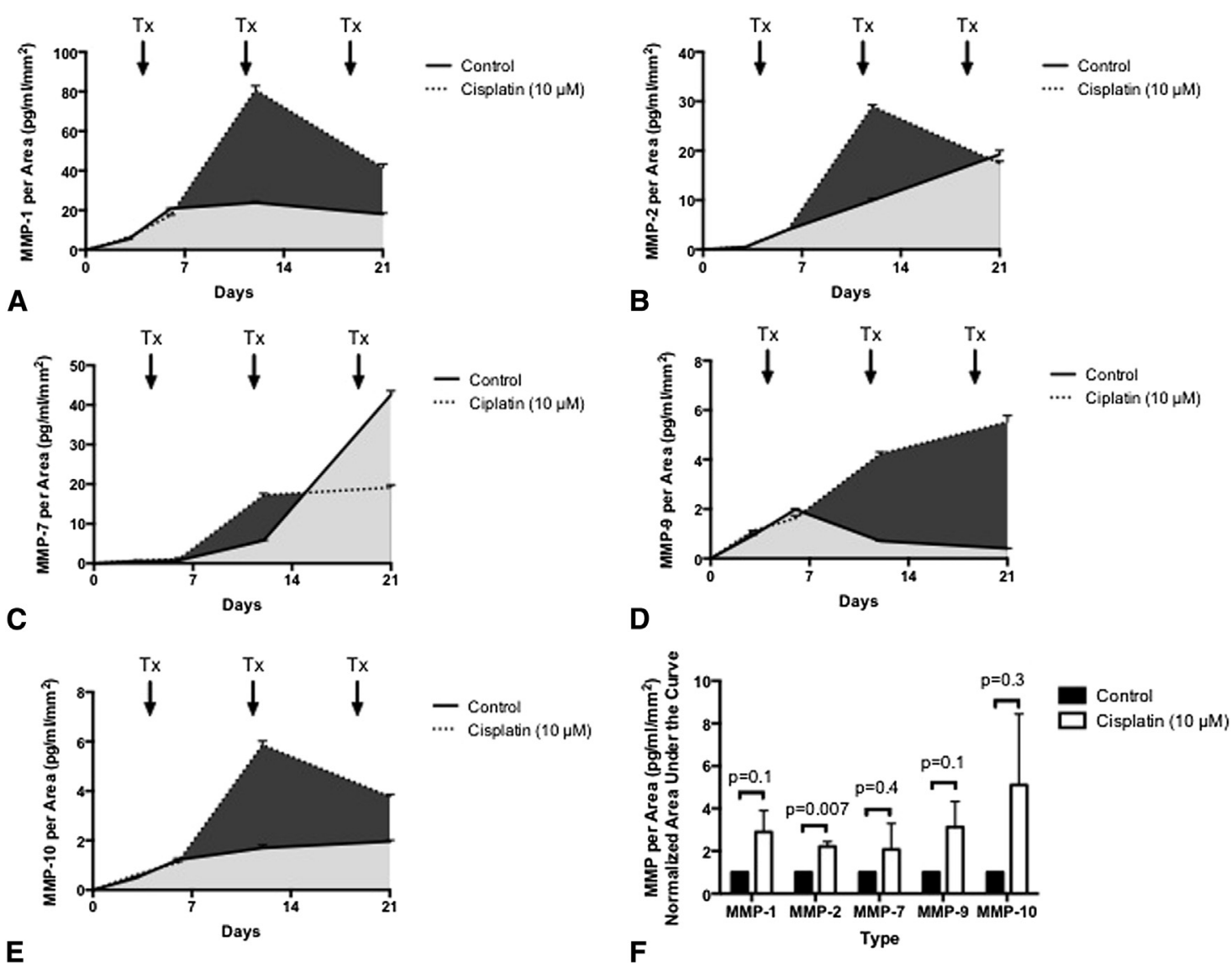

FIGURE 5. Matrix metalloproteinase (MMP) production per tumor area in the 4D model with cisplatin treatment. A-D, MMP-1 (A), MMP-2 (B), MMP-7 (C), MMP-9 (D), MMP-10 (E) level per tumor area for the 4D model seeded with H1299 cells treated with $10 \mu \mathrm{M}$ cisplatin on days 4, 11 , and 18 compared with the control group. F, The normalized average area under the curve for MMP-2 production per tumor area was significantly higher for the 4D model seeded with A549, H1299, and HCC827 cells compared with the control group ( $P=.007)$; there was no significant difference for MMP-1, MMP-7, MMP-9, and MMP-10. $T x$, Treatment.

scan, regression of lung cancer nodules is a hallmark response to lung cancer treatment ${ }^{16}$ and our $4 \mathrm{D}$ lung model also shows nodule regression in response to treatment. The analysis of the tissue confirms this with lower proliferation (Ki-67) and higher cell death (caspase-3) with a more immediate effect with $50 \mu \mathrm{M}$ cisplatin with most cells showing necrosis 4 days after treatment; it took 10 days with $10 \mu \mathrm{M}$ cisplatin. After 2 days of treatment with 50 $\mu \mathrm{M}$ cisplatin, there is a significant increase in live CTCs per tumor area, whereas there is an increase on days 4 and 6 after treatment with $10 \mu \mathrm{M}$ cisplatin, both of which dissipated with necrosis of the primary tumor. We postulated that cisplatin might cause the cells in the nodule to become CTCs. This concept was tested by intermittent treatment of physiologic concentration of cisplatin. We found that for all 4 cell lines that were grown in the model, there were significant decreases in the tumor size and significant increases in CTCs per tumor area when the 4D model with human lung cancer cell lines was treated with cisplatin. This may model the formation of resistant metastatic lesions in patients with lung cancer. In patients with lung cancer, cisplatin greatly affects the primary tumor size. However, this may lead to the formation of CTCs that are resistant to cisplatin treatment and allow for the development of metastatic lesions that are resistant to cisplatin.

This increase in CTC production per tumor area is correlated with an increase in MMP-2, a protein involved in the breakdown of the extracellular matrix. It is known to break down type IV collagen, which is the major structural component of basement membrane. ${ }^{17}$ MMP-2 has been found at higher levels in serum in patients with lung cancer compared with benign and control groups. ${ }^{18}$ It has also been shown to be a more sensitive predictor than MMP-9 for lung cancer progression, metastasis, and survival. ${ }^{19}$ MMP-2 may be involved in the release of CTCs from the primary tumor when it is treated with cisplatin.

The limitation of the current study is that these findings have yet to be confirmed in patients with lung cancer. We postulate that when patients with lung cancer are given 
cisplatin, there will be a significant increase in CTCs per tumor area and that these CTCs will be more resistant to cisplatin. We plan to investigate our hypothesis as we improve our ability to isolate CTCs from patients with lung cancer.

To our knowledge, the current study is the first to show the effect of cisplatin treatment on perfusable nodules and the selection of cisplatin-resistant CTCs. The 4D lung model is not likely to replace the current in vivo mouse models, but it will provide additional information through its ability to control many aspects of tumor growth and development and will allow improved understanding of the mechanisms of action of various anticancer drugs. Ultimately, the 4D model could potentially provide an alternative approach to study drug efficacy, mechanisms of resistance, and molecular insights into the treatment of non-small cell lung cancer.

\section{References}

1. Siegel R, Naishadham D, Jemal A. Cancer statistics, 2013. CA Cancer J Clin. 2013;63:11-30.

2. Jemal A, Siegel R, Xu J, Ward E. Cancer statistics, 2010. CA Cancer J Clin. 2010;60:277-300

3. Petrelli F, Coinu A, Cabiddu M, Ghilardi M, Ardine M, Barni S. Platinum rechallenge in patients with advanced NSCLC: a pooled analysis. Lung Cancer. 2013;81:337-42.

4. Shaw GL, Gazdar AF, Phelps R, Steinberg SM, Linnoila RI, Johnson BE, et al. Correlation of in vitro drug sensitivity testing results with response to chemotherapy and survival: comparison of non-small cell lung cancer and small cell lung cancer. J Cell Biochem Suppl. 1996;24:173-85.

5. Vescio RA, Connors KM, Kubota T, Hoffman RM. Correlation of histology and drug response of human tumors grown in native-state three-dimensional histoculture and in nude mice. Proc Natl Acad Sci U S A. 1991;88:5163-6.

6. Ott HC, Clippinger B, Conrad C, Schuetz C, Pomerantseva I, Ikonomou L, et al. Regeneration and orthotopic transplantation of a bioartificial lung. Nat Med. 2010;16:927-33.

7. Mishra DK, Thrall MJ, Baird BN, Ott HC, Blackmon SH, Kurie JM, et al. Human lung cancer cells grown on acellular rat lung matrix create perfusable tumor nodules. Ann Thorac Surg. 2012;93:1075-81.

8. Mishra DK, Sakamoto JH, Thrall MJ, Baird BN, Blackmon SH, Ferrari M, et al. Human lung cancer cells grown in an ex vivo 3D lung model produce matrix metalloproteinases not produced in 2D culture. PLoS one. 2012;7:e45308.

9. Mishra DK, Creighton CJ, Zhang Y, Gibbons DL, Kurie JM, Kim MP. Gene expression profile of A549 cells from tissue of 4D model predicts poor prognosis in lung cancer patients. Int J Cancer. 2014;134:789-98.

10. Giaccone G, Battey J, Gazdar AF, Oie H, Draoui M, Moody TW. Neuromedin B is present in lung cancer cell lines. Cancer Res. 1992;52:2732s-6s.

11. Giard DJ, Aaronson SA, Todaro GJ, Arnstein P, Kersey JH, Dosik H, et al. In vitro cultivation of human tumors: establishment of cell lines derived from a series of solid tumors. J Natl Cancer Inst. 1973;51:1417-23.

12. Girard L, Zochbauer-Muller S, Virmani AK, Gazdar AF, Minna JD. Genome-wide allelotyping of lung cancer identifies new regions of allelic loss, differences between small cell lung cancer and non-small cell lung cancer, and loci clustering. Cancer Res. 2000;60:4894-906.

13. Himmelstein KJ, Patton TF, Belt RJ, Taylor S, Repta AJ, Sternson LA. Clinical kinetics on intact cisplatin and some related species. Clin Pharmacol Ther. 1981; 29:658-64.

14. Kashkin KN, Musatkina EA, Komelkov AV, Tonevitsky EA, Sakharov DA, Vinogradova TV, et al. Genes potentially associated with cisplatin resistance of lung cancer cells. Dokl Biochem Biophys. 2011;438:147-50.

15. Shen H, Perez RE, Davaadelger B, Maki CG. Two $4 \mathrm{~N}$ cell-cycle arrests contribute to cisplatin-resistance. PLoS one. 2013;8:e59848.

16. Dillman RO, Herndon J, Seagren SL, Eaton WL Jr, Green MR. Improved survival in stage III non-small-cell lung cancer: seven-year follow-up of cancer and leukemia group B (CALGB) 8433 trial. J Natl Cancer Inst. 1996;88:1210-5.
17. Ortega N, Werb Z. New functional roles for non-collagenous domains of basement membrane collagens. J Cell Sci. 2002;115:4201-14.

18. Ali-Labib R, Louka ML, Galal IH, Tarek M. Evaluation of matrix metalloproteinase-2 in lung cancer. Proteomics Clin Appl. 2014;8:251-7.

19. Guo CB, Wang S, Deng C, Zhang DL, Wang FL, Jin XQ. Relationship between matrix metalloproteinase 2 and lung cancer progression. Mol Diagn Ther. 2007; 11:183-92.

\section{Discussion}

Dr Chadrick E. Denlinger (Charleston, SC). Congratulations, $\mathrm{Dr} \mathrm{Kim}$, on a very nice presentation of your interesting model. This lung cancer model, which your group has published on previously, is unique and potentially very important because of the limitations inherent with traditional lung cancer cell line models. The advantage of cell lines is that they are relatively inexpensive and they are very reproducible, but I think some of the limitation is that I am not sure that cancer cells grown on Petri dishes are really representative of actual cancers in humans, and this is a point you made in your presentation and in your manuscript.

The data presented here represent 4 different cell lines grown in your $4 \mathrm{D}$ model, which leads me to my first question. Even though you have shown here and previously that your cancer cell lines behave differently in the rat lung scaffold compared with standard tissue culture, how do you know they are more representative of reality, meaning actual cancers, than standard tissue culture?

Dr Kim. We have done several different experiments to try and answer that question. First, if you take a cell line derived from a cancer with a papillary phenotype and put it into our model, it recaptures the papillary phenotype on hematoxylin and eosin. Even though on our culture it looks like it is single cells that are growing, in our model it kind of recaptures the pathology that you see in a patient with lung cancer. The other thing we have done is we compared the MMP production in a $2 \mathrm{D}$ versus our $4 \mathrm{D}$ model and the MMP production of the 4D model mimics the production in patients with cancer. Lastly, we compared gene expression profiles with a 4D model versus a 3D model, where the 3D model is the cells that are grown on artificial Matrigel, and there seems to be better correlation in terms of patient survival with the 4D model compared with the 3D model. So there is some evidence suggesting that this model might be better mimicked than what we see in 2D and 3D cultures.

Dr Denlinger. Have you attempted to grow primary cancer cell lines in your model taken directly from patients?

Dr Kim. We have attempted to grow primary cancer cells. In order to capture this kind of biology, we need about 25 million cells to capture in this 14-day or 21-day time period. The problem that we are having with trying to capture from a primary tumor from a patient is the number of cells that we can easily put into our model. We have not been successful yet, but I think that is one of the aims of the laboratory.

Dr Denlinger. Interesting. What you are describing as CTCs in this model does sound rather analogous to cancer stem cells or cells that have undergone epithelial-mesenchymal transition. Have you characterized your circulating tumor cells for markers like CD133 or CD44 that would define them as the cancer stem cells?

Dr Kim. We have not done that experiment yet. I think that is one of the things that we are going to be looking at in future studies. We have done some gene expression profiles, which 
show that there is a decrease in epithelial markers and an increase in mesenchymal markers.

Dr Denlinger. You showed that your circulating tumor cells are resistant to cisplatin. That is initially. If you continue to grow those cells for several passages in tissue culture, do they retain their morphology or their behavior and are cisplatin-resistant or do they revert back to your standard H460s or H1299s?

Dr Kim. We have not done that experiment yet. I think the CTCs that we are seeing is just a phase of this tumor development.
In patients we do not really see the CTCs going back on plastic and growing. We have not done that experiment because I am not sure how that is going to translate exactly into patients. What we have been trying to do is take these CTCs and put them back into our model, thinking that it would be creating a metastasis model and see if those metastatic lesions are more resistant to CTCs, which would be more analogous to what is happening in patients.

Dr Denlinger. Thank you and congratulations. 